\title{
Establishing nanomedicine
}

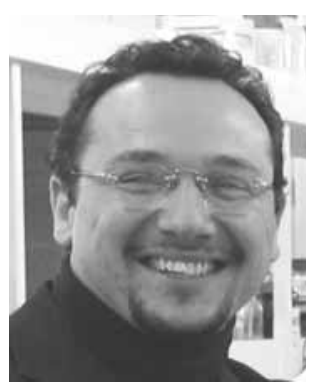

Kotas Kostardos

Senior Editor

$\mathrm{N}$ anomedicine Laboratory, Centre for D rug D elivery Research, U niversity of London, The School of Pharmacy, 29-39 Brunswick Square, London, WCIN IAX, UK Tel.: +44 2077535861 ; Fax: +44 207753 5942; E-mail: kostas.kostarelos@ pharmacy.ac.uk
'What has helped tremend ously in the gradual recognition of the na nomedic ine field that we are witnessing today is the consistent support for all aspects and a pplic ations of na notechnology researc $h$ from public so urc es of fund ing.'

$\mathrm{N}$ anomedicine is claimed by many to be a brainchild of the past, from Richard Feynman's quotes that are commonly cited as the origin of all 'nano', to science fiction movies of the 1950s and 1960s. $\mathrm{H}$ owever, after a simple investigation in the two most popular scientific publication search engines (PubM ed and ISI Web of Knowledge), one immediately realizes that 'nanomedicine' as a recognized scientific term is clearly a child of the 2000s. Encouragingly enough for us involved in this field, even though the number of citations using the word 'nanomedicine' as a keyword in both search engines is still surprisingly small, there is a healthy increase in the frequency of the term's use (Figre1). Such very obvious and unsophisticated analysis is thought to indicate the following two points: 'nanomedicine' is a young term that is still finding its way in the mainstream of scientific literature; and the steep increase in the popularity of the term means that the field is growing rapidly al ong with acceptance of its ensuing concepts and technologies.

What has helped tremendously in the gradual recognition of the nanomedicine field that we are witnessing today is the consistent support for all aspects and applications of nanotechnology research from public sources of funding. The USA, the EU and Japan have all pledged significant amounts of 'start-up' investment for nanotechnology and nanomedicine. It is difficult to assess accurately the amount of funding dedicated exclusively to nanomedicine, since a lot of the nanotechnologies under development are aimed ultimately at the development of either diagnostic or therapeutic products, even though the clinical applicability of such emerging technologies is not readily apparent. Nevertheless, specific programs of funding announced and titled as 'nanomedicine funding calls' were first awarded in 2005 through the $\mathrm{C}$ ancer $\mathrm{N}$ anotechnology Excellence Awards at the $\mathrm{N}$ ational $\mathrm{C}$ ancer Institute and more centers are being established and funded this year. Today in the USA, most of the National Institutes at the $\mathrm{NIH}$ have already announced or awarded dedicated programs of research in nanomedicine [1,2], whereas nanomedicine features as part of the $\mathrm{N} \mathrm{IH}$ Roadmap for M edical Research with the $\mathrm{N}$ ational Eye Institute responsible for coordination of the $\mathrm{NIH}$ nanomedicine initiatives [3].

Interestingly, the $\mathrm{N} \mathrm{IH}$ is looking at the nanomedicine field as a rich source of new technologies with a medium- to long-term perspective. That is somewhat different to the European perspective, where a more industry-led, early commercialization approach is sought after. The field in Europe is even more dissipate than in the USA owing to the difficulty in coordinating work at the national level, but it is expected to start consolidating through the coordinated efforts of the European Commission and the establishment of the European Technology Platform in nanomedicine [4]. The 7th Framework Programme, which is the main instrument of research funding at the

Figure 1. Citation numbers using the search term 'nanomedicine' in two popular scientific publication search engines.

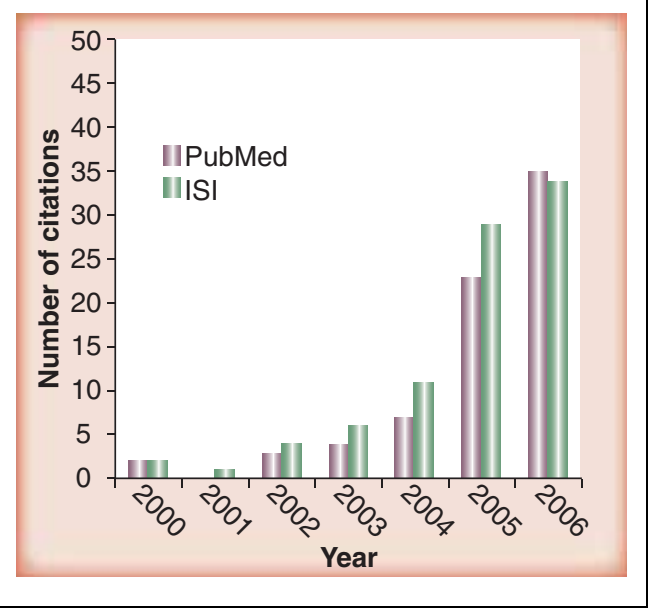


European Commission level, will contain nanomedicine as a strategic theme and specific calls on nanomedicine are expected.

'Quite a few big pharma groupsare attracted to the conceptsand tec hnologies that na nomedic ine presents, however, it is still generally considered to be at too early a stage and of high risk.'

D espite the committed approach and relative long-term deliverables expected from public funding agencies, the pharmaceutical industry is still apprehensive of nanomedicine. Q uite a few big pharma groups are attracted to the concepts and technologies that nanomedicine presents, however, it is still generally considered to be at too early a stage and of high risk. M ost of the large pharmaceutical companies are consolidating their 'nanomedicinal' patent portfolios and the technologies that best fit their existing markets in these early days. The stance of venture capital investment is also one of cautious interest before making sure that nanomedicine is not another 'bubble' waiting to burst but a real opportunity. The estimated investment from venture capital al ready expended in this decade is approximately US\$900 million. Confidence in the private investment sector for nanomedicine, either large industry or venture capital, will be one of the determining factors in bringing forward all of the budding technologies that public investment is initiating through its current activities, so moving them closer to the clinic and the patient.
Figure 2. Screen-shot of an animated educational 'nano' video for children in Japan.

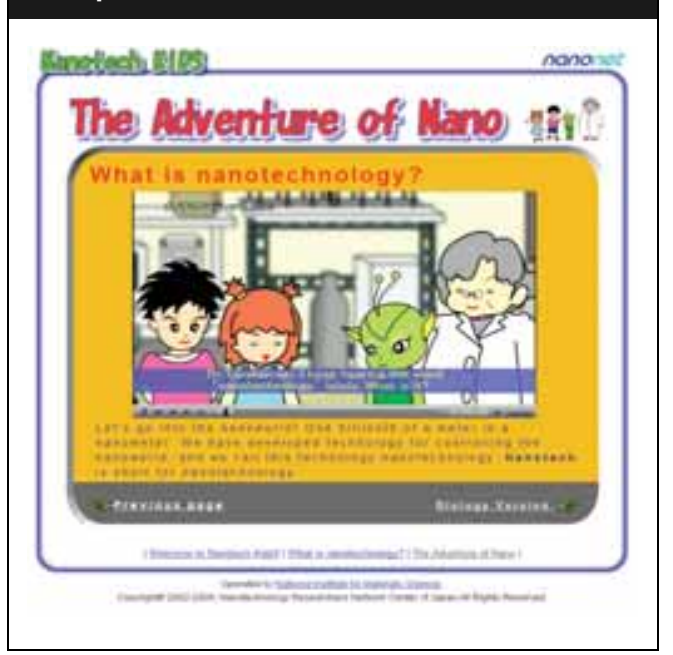

W ith $\mathrm{N}$ anomedicine, we are expecting to act as a valuable platform for discussion, exchange of ideas and stage to showcase all of the nanomedicine activities and their interesting products in every relevant form: technical, clinical, ethical and societal. The journey with nanomedicine promises to be exciting, captivating the public's attention and eventually leading to the most sophisticated tools against disease ever available to humankind. Let's see what the next generation will think of our achievements. For the moment, all we have to do is educate the public from as young an age as possible (similar to the animated educational video produced recently by the Japanese M inistry of Science \& Technology [5]) (Figre2) and try with best intentions.
Bibliography

1. N ational Cancer Institute: $\mathrm{N} \mathrm{Cl}$ Alliance for $\mathrm{N}$ anotechnology in cancer http://nano.cancer.gov

2. N ational H uman $\mathrm{G}$ enome Research Institute: N anomedicine www.genome.gov/11508736
3. N IH Roadmap for M edical Research: $\mathrm{N}$ anomedicine http://nihroadmap.nih.gov/nanomedicine/ index.asp

4. European Technology Platform: Nanomedicine http://cordis.europa.eu/nanotechnology/ nanomedicine.htm
5. Nanotech Kids www.nanonet.go.jp/english/kids/video/ index2.html 